\title{
General Minimal Flavor Violation
}

\author{
Alexander L. Kagan, ${ }^{1}$ Gilad Perez, ${ }^{2,3}$ Tomer Volansky, ${ }^{4}$ and Jure Zupan ${ }^{5,6}$ \\ ${ }^{1}$ Department of Physics, University of Cincinnati, Cincinnati, Ohio 45221, USA \\ ${ }^{2}$ Department of Particle Physics, Weizmann Institute of Science, Rehovot 76100, Israel \\ ${ }^{3}$ YITP, Stony Brook University, Stony Brook, NY 11794-3840, USA \\ ${ }^{4}$ School of Natural Sciences, Institute for Advanced Study, Princeton, NJ 08540 \\ ${ }^{5}$ Theory Division, Department of Physics, CERN, CH-1211 Geneva 23, Switzerland \\ ${ }^{6}$ Faculty of mathematics and physics, University of Ljubljana, Jadranska 19, 1000 Ljubljana, Slovenia
}

\begin{abstract}
A model independent study of the minimal flavor violation (MFV) framework is presented, where the only sources of flavor breaking at low energy are the up and down Yukawa matrices. Two limits are identified for the Yukawa coupling expansion: linear MFV, where it is truncated at the leading terms, and nonlinear MFV, where such a truncation is not possible due to large third generation Yukawa couplings. These are then resummed to all orders using non-linear $\sigma$-model techniques familiar from models of collective breaking. Generically, flavor diagonal CP violating (CPV) sources in the UV can induce $O(1) \mathrm{CPV}$ in processes involving third generation quarks. Due to a residual $\mathrm{U}(2)$ symmetry, the extra CPV in $B_{d}-\bar{B}_{d}$ mixing is bounded by CPV in $B_{s}-\bar{B}_{s}$ mixing. If operators with right-handed light quarks are subdominant, the extra CPV is equal in the two systems, and is negligible in processes involving only the first two generations. We find large enhancements in the up type sector, both in CPV in $D-\bar{D}$ mixing and in top flavor violation.
\end{abstract}

Introduction. Precision flavor and $\mathrm{CP}$ violation measurements provide very strong constraints on models of new physics (NP) beyond the Standard Model (SM). For instance, $\epsilon_{K}$ constrains the scale of maximally flavor violating NP to be $\gtrsim 10^{4} \mathrm{TeV}$. Therefore, $\mathrm{TeV}$ scale NP which stabilizes the electroweak scale and is accessible at the LHC has to have a highly non generic flavor structure.

The tension with precision flavor tests is relaxed if the SM Yukawa matrices are the only source of flavor breaking, even in the presence of new particles and interactions [1-3]. This hypothesis goes under the name of Minimal Flavor Violation (MFV). Sometimes additional assumptions are made - that the SM Yukawa couplings are also the only source of $\mathrm{CP}$ violation (CPV), e.g. in [1], or that NP does not change the Lorentz structure of the effective weak hamiltonian [4]. We will not make these assumptions, but will discuss their consequences below.

A useful language for discussing MFV was introduced in [1]. It relies on the observation that for vanishing Yukawa couplings the SM has an enhanced global symmetry. Focusing on the quark sector this is

$$
\mathcal{G}^{\mathrm{SM}}=U(3)_{Q} \times U(3)_{u} \times U(3)_{d}
$$

where $Q, u, d$ stand for quark doublets and up and down type quark singlets respectively. The SM Yukawa couplings

$$
H_{u} \bar{Q}_{L} Y_{u} u_{R}+H_{d} \bar{Q}_{L} Y_{d} d_{R}
$$

are formally invariant under $\mathcal{G}^{\mathrm{SM}}$, if the Yukawa matrices are promoted to spurions that transform as $Y_{u, d}^{\prime}=$ $V_{Q} Y_{u, d} V_{u, d}^{\dagger}$, while the quark fields are in the fundamental representations, $\left(Q^{\prime}, u^{\prime}, d^{\prime}\right)=V_{Q, u, d}(Q, u, d)$. Weak scale NP models are then of the MFV class if they are formally invariant under $\mathcal{G}^{\mathrm{SM}}$, when treating the SM Yukawa couplings as spurions. Similarly, the low energy flavor observables are formally invariant under $\mathcal{G}^{\mathrm{SM}}$. Practically, this means that only certain insertions of Yukawa couplings are allowed in the quark bilinears. For example, in $\bar{Q} Q$ bilinears insertions such as $\bar{Q}\left(Y_{u} Y_{u}^{\dagger}\right)^{n} Q$ are allowed, while $\bar{Q} Y_{d}^{\dagger}\left(Y_{u} Y_{u}^{\dagger}\right)^{n} Q$ are not.

The above definition of MFV is only useful if flavor invariant operators such as $\bar{Q} f\left(\epsilon_{u} Y_{u}, \epsilon_{d} Y_{d}\right) Q$ can be expanded in powers of $Y_{u, d}$. In the large $\tan \beta$ limit both $Y_{u}$ and $Y_{d}$ have $O(1)$ eigenvalues $y_{t, b}$. The convergence radius is then given by the size of $\epsilon_{u, d}$. We distinguish between two limiting cases

- Linear MFV (LMFV): $\epsilon_{u, d} \ll 1$ and the dominant flavor breaking effects are captured by the lowest order polynomials of $Y_{u, d}$.

- Non-linear MFV (NLMFV): $\epsilon_{u, d} \sim O(1)$, higher powers of $Y_{u, d}$ are important, and a truncated expansion in $y_{t, b}$ is not possible.

Examples of NLMFV are: low energy supersymmetric models in which large $\tan \beta$ effects need to be resummed (large $\epsilon_{d}$ ), and models obeying MFV at a UV scale $\Lambda_{F} \gg \mu_{W}$, where large $\epsilon_{u, d} \propto \log \left(\mu_{W} / \Lambda_{F}\right)$ are generated from sizable anomalous dimensions in the renormalization group running [5]. Another example is warped extra dimension models with alignment [6], in cases where right handed up-quark currents are subdominant.

In this letter we show that even in NLMFV there is a systematic expansion in small quantities, $V_{t d}, V_{t s}$, and light quark masses, while resumming in $y_{t}, y_{b} \sim$ $O(1)$. This is achieved via a non-linear $\sigma$-model-like parametrization. Namely, in the limit of vanishing weak gauge coupling (or $m_{W} \rightarrow \infty$ ), $U(3)_{Q}$ is enhanced to $U(3)_{Q^{u}} \times U(3)_{Q^{d}}$. The two groups are broken down 
to $U(2) \times U(1)$ by large third generation eigenvalues in $Y_{u, d} Y_{u, d}^{\dagger}$, so that the low energy theory is described by a $[U(3) / U(2) \times U(1)]^{2}$ non-linear $\sigma$-model. Flavor violation arises due to the misalignment of $Y_{u}$ and $Y_{d}$, given by $V_{t d}$ and $V_{t s}$ once the weak interaction is turned on. We can then prove with complete generality that in MFV: (i) extra CPV can only arise from flavor diagonal CPV sources in the UV theory; (ii) the extra $\mathrm{CP}$ phases in $B_{s}-\bar{B}_{s}$ mixing provide an upper bound on the amount of CPV in $B_{d}-\bar{B}_{d}$ mixing; (iii) if operators containing right-handed light quarks are subdominant then the extra $\mathrm{CPV}$ is equal in the two systems, and is negligible in $2 \rightarrow 1$ transitions. Conversely, these operators can break the correlation between $\mathrm{CPV}$ in the $B_{s}$ and $B_{d}$ systems, and can induce significant new $\mathrm{CPV}$ in $\epsilon_{K}$. Combinations of observables which are sensitive to LMFV vs. NLMFV are also identified. Another non-linear parameterization of MFV was presented in [7]. We focus on exploiting the general control obtained by our formalism in order to study its model independent implications. A modification of the formalism is needed for $y_{b} \ll 1$, as discussed below.

Formalism. To realize $\mathcal{G}^{\mathrm{SM}}$ non-linearly, we promote the Yukawa matrices to spurions, with the transformation properties given below Eq. (2). These flavor transformations are broken once the Yukawa couplings obtain their background values. The eigenvalues of the latter are hierarchical and the two matrices are approximately aligned. We therefore take $Y_{u} \sim \operatorname{diag}\left(0,0, y_{t}\right)$ and $Y_{d} \sim$ $\operatorname{diag}\left(0,0, y_{b}\right)$. The breaking of the flavor group is dominated by the top and bottom Yukawa couplings which break it down to $\mathcal{H}^{\mathrm{SM}}=U(2)_{Q} \times U(2)_{u} \times U(2)_{d} \times U(1)_{3}$.

The broken symmetry generators live in $\mathcal{G}^{\mathrm{SM}} / \mathcal{H}^{\mathrm{SM}}$ cosets. It is useful to factor them out of the Yukawa matrices. We thus use the parameterization

$$
Y_{u, d}=e^{i \hat{\rho}_{Q}} e^{ \pm i \hat{\chi} / 2} \tilde{Y}_{u, d} e^{-i \hat{\rho}_{u, d}}
$$

where the reduced Yukawa spurions, $\tilde{Y}_{u, d}$, are

$$
\tilde{Y}_{u, d}=\left(\begin{array}{cc}
\phi_{u, d} & 0 \\
0 & y_{t, b}
\end{array}\right) .
$$

Here $\phi_{u, d}$ are $2 \times 2$ complex spurions, while $\hat{\chi}$ and $\hat{\rho}_{i}$, $i=Q, u, d$, are the $3 \times 3$ matrices spanned by the broken generators. Explicitly,

$$
\hat{\chi}=\left(\begin{array}{cc}
0 & \chi \\
\chi^{\dagger} & 0
\end{array}\right), \quad \hat{\rho}_{i}=\left(\begin{array}{cc}
0 & \rho_{i} \\
\rho_{i}^{\dagger} & \theta_{i}
\end{array}\right), \quad i=Q, u, d,
$$

where $\chi$ and $\rho_{i}$ are two dimensional vectors. The $\rho_{i}$ shift under the broken generators and therefore play the role of spurion "Goldstone bosons". Thus the $\rho_{i}$ have no physical significance. $\chi$, on the other hand, parametrizes the misalignment of the up and down Yukawa couplings and will therefore correspond to $V_{t d}$ and $V_{t s}$ in the low energy effective theory [see Eq. (12)].
Under the flavor group the above spurions transform as,

$$
e^{i \hat{\rho}_{i}^{\prime}}=V_{i} e^{i \hat{\rho}_{i}} U_{i}^{\dagger}, \quad e^{i \hat{\chi}^{\prime}}=U_{Q} e^{i \hat{\chi}} U_{Q}^{\dagger}, \quad \tilde{Y}_{i}^{\prime}=U_{Q} \tilde{Y}_{i} U_{i}^{\dagger} .
$$

Here $U_{i}=U_{i}\left(V_{i}, \hat{\rho}_{i}\right)$ are (reducible) unitary representations of the unbroken flavor subgroup $U(2)_{i} \times U(1)_{3}$,

$$
U_{i}=\left(\begin{array}{cc}
U_{i}^{2 \times 2} & 0 \\
0 & e^{i \varphi_{3}}
\end{array}\right), \quad i=Q, u, d .
$$

For $V_{i} \in \mathcal{H}^{\mathrm{SM}}, U_{i}=V_{i}$. Otherwise the $U_{i}$ depend on the broken generators and $\hat{\rho}_{i}$. They form a nonlinear realization of the full flavor group. In particular, Eq. (6) defines $U_{i}\left(V_{i}, \hat{\rho}_{i}\right)$ by requiring that $\hat{\rho}_{i}^{\prime}$ is of the same form as $\hat{\rho}_{i}$, Eq. (5). Consequently $\hat{\rho}_{i}$ is shifted under $\mathcal{G}^{\mathrm{SM}} / \mathcal{H}^{\mathrm{SM}}$ and can be set to a convenient value as discussed below. Under $\mathcal{H}^{\mathrm{SM}}, \chi\left[\rho_{i}\right]$ are fundamentals of $U(2)_{Q}\left[U(2)_{i}\right]$ carrying charge -1 under the $U(1)_{3}$, while $\phi_{u, d}$ are bi-fundamentals of $U(2)_{Q} \times U(2)_{u, d}$.

As a final step we also redefine the quark fields by moding out the "Goldstone spurions",

$$
\begin{array}{cc}
\tilde{u}_{L}=e^{-i \hat{\chi} / 2} e^{-i \hat{\rho}_{Q}} u_{L}, & \tilde{d}_{L}=e^{i \hat{\chi} / 2} e^{-i \hat{\rho}_{Q}} d_{L}, \\
\tilde{u}_{R}=e^{-i \hat{\rho}_{u}} u_{R}, & \tilde{d}_{R}=e^{-i \hat{\rho}_{d}} d_{R} .
\end{array}
$$

The latter form reducible representations of $\mathcal{H}^{\mathrm{SM}}$. Concentrating here and below on the down sector we therefore define $\tilde{d}_{L, R}=\left(\tilde{d}_{L, R}^{(2)}, 0\right)+\left(0, \tilde{b}_{L, R}\right)$. Under flavor transformations $\tilde{d}_{L}^{(2) \prime}=U_{Q}^{2 \times 2} \tilde{d}_{L}^{(2)}$ and $\tilde{b}_{L}^{\prime}=\exp \left(i \varphi_{3}\right) \tilde{b}_{L}$. A similar definition can be made for the up quarks.

With the redefinitions above, invariance under the full flavor group is captured by the invariance under the unbroken flavor subgroup $\mathcal{H}^{\mathrm{SM}}$ [8]. Thus, NLMFV can be described without loss of generality as a formally $\mathcal{H}^{\mathrm{SM}}$ invariant expansion in $\phi_{u, d}, \chi$. This is a straightforward generalization of the known effective field theory description of spontaneous symmetry breaking [8]. The only difference in our case is that $Y_{u, d}$ are not aligned, as manifested by $\chi \neq 0$. Since the background field values of the relevant spurions are small, we can expand in them.

We are now in a position to write down the flavor structures of quark bilinears from which low energy flavor observables can be constructed. We work to leading order in the spurions that break $\mathcal{H}^{\mathrm{SM}}$, but to all orders in the top and bottom Yukawa couplings. Beginning with the left-left (LL) bilinears, to second order in $\chi, \phi_{u, d}$ one finds (omitting gauge and Lorentz indices)

$$
\begin{gathered}
\overline{\tilde{b}}_{L} \tilde{b}_{L}, \quad \overline{\tilde{d}_{L}^{(2)}} \tilde{d}_{L}^{(2)}, \quad \overline{\tilde{d}}_{L}^{(2)} \phi_{u} \phi_{u}^{\dagger} \tilde{d}_{L}^{(2)}, \\
\overline{\tilde{d}}_{L}^{(2)} \chi \tilde{b}_{L}, \quad \overline{\tilde{b}}_{L} \chi^{\dagger} \chi \tilde{b}_{L}, \quad \overline{\tilde{d}_{L}^{(2)}} \chi \chi^{\dagger} \tilde{d}_{L}^{(2)} .
\end{gathered}
$$

The first two bilinears in Eq. (10) are diagonal in the down-quark mass basis and do not induce flavor violation. In this basis the Yukawa couplings take the form $Y_{u}=V_{\mathrm{CKM}}^{\dagger} \operatorname{diag}\left(m_{u}, m_{c}, m_{t}\right), Y_{d}=\operatorname{diag}\left(m_{d}, m_{s}, m_{b}\right)$. 
This corresponds to spurions taking the background values $\rho_{Q}=\chi / 2, \hat{\rho}_{u, d}=0, \phi_{d}=\operatorname{diag}\left(m_{d}, m_{s}\right) / m_{b}$, while flavor violation is induced via

$$
\chi^{\dagger}=i\left(V_{t d}, V_{t s}\right), \quad \phi_{u}=V_{\mathrm{CKM}}^{(2) \dagger} \operatorname{diag}\left(\frac{m_{u}}{m_{t}}, \frac{m_{c}}{m_{t}}\right) .
$$

$V_{\text {CKM }}^{(2)}$ stands for a two generation CKM matrix. In terms of $\lambda=\sin \theta_{C} \simeq 0.23$, the flavor violating spurions scale as $\chi \sim\left(\lambda^{3}, \lambda^{2}\right),\left(\phi_{u}\right)_{12} \sim \lambda^{5}$. Note that the redefined down quark fields, Eqs. $(8,9)$, coincide with the masseigenstate basis, $\tilde{d}_{L, R}=d_{L, R}$, for the above choice of spurion background values.

The left-right (LR) and right-right (RR) bilinears which contribute to flavor mixing are in turn (at leading order in $\chi, \phi_{u, d}$ spurions),

$$
\begin{gathered}
\overline{\tilde{d}_{L}^{(2)}} \chi \tilde{b}_{R}, \quad \overline{\tilde{d}_{L}^{(2)}} \chi \chi^{\dagger} \phi_{d} \tilde{d}_{R}^{(2)}, \quad \overline{\tilde{b}}_{L} \chi^{\dagger} \phi_{d} \tilde{d}_{R}^{(2)}, \\
\overline{\tilde{d}_{R}^{(2)}} \phi_{d}^{\dagger} \chi \tilde{b}_{R}, \quad \overline{\tilde{d}_{R}^{(2)}} \phi_{d}^{\dagger} \chi \chi^{\dagger} \phi_{d} \tilde{d}_{R}^{(2)} .
\end{gathered}
$$

To make contact with the more familiar MFV notation, consider down quark flavor violation from LL bilinears. We can then expand in the Yukawa couplings,

$\bar{Q}\left[a_{1} Y_{u} Y_{u}^{\dagger}+a_{2}\left(Y_{u} Y_{u}^{\dagger}\right)^{2}\right] Q+\left[b_{2} \bar{Q} Y_{u} Y_{u}^{\dagger} Y_{d} Y_{d}^{\dagger} Q+h . c.\right]+\cdots$,

with $a_{1,2}=O\left(\epsilon_{u}^{2,4}\right), b_{2}=O\left(\epsilon_{u}^{2} \epsilon_{d}^{2}\right)$. Following the discussion in the Introduction, the LMFV limit corresponds to $a_{1} \gg a_{2}, b_{2}$, and the NLMFV limit to $a_{1} \sim a_{2} \sim b_{2}$. While $a_{1,2}$ are real, the third operator in Eq. (15) is not Hermitian and $b_{2}$ can be complex [9], introducing a new $\mathrm{CP}$ violating phase beyond the SM phase. The leading flavor violating terms in Eq. (15) for the down quarks are

$\bar{d}_{L}^{i}\left[\left(a_{1}+a_{2} y_{t}^{2}\right) \xi_{i j}^{t}+a_{1} \xi_{i j}^{c}\right] d_{L}^{j}+\left[b_{2} y_{b}^{2} \bar{d}_{L}^{i} \xi_{i b}^{t} b_{L}+h . c.\right]=$

$c_{b}\left(\overline{\tilde{d}_{L}^{(2)}} \chi \tilde{b}_{L}+h . c\right)+c_{t} \overline{\tilde{d}_{L}^{(2)}} \chi \chi^{\dagger} \tilde{d}_{L}^{(2)}+c_{c} \overline{\tilde{d}_{L}^{(2)}} \phi_{u} \phi_{u}^{\dagger} \tilde{d}_{L}^{(2)}$,

where $\xi_{i j}^{k}=y_{k}^{2} V_{k i}^{*} V_{k j}$ with $i \neq j$. On the RHS we have used the general parameterization in Eqs. $(10,11)$ with $c_{b} \simeq\left(a_{1} y_{t}^{2}+a_{2} y_{t}^{4}+b_{2} y_{b}^{2}\right), c_{t} \simeq a_{1} y_{t}^{2}+a_{2} y_{t}^{4}$ and $c_{c} \simeq a_{1}$ to leading order. The contribution of the $c_{c}$ bilinear in flavor changing transitions is $O(1 \%)$ compared to the $c_{t}$ bilinear, and can be neglected in practice.

LMFV vs. NLMFV. A novel feature of NLMFV is the potential for observable CPV from right-handed currents, to which we return below. Other important distinctions can be readily understood from Eq. (16). In NLMFV (with large $\tan \beta$ ) the extra flavor diagonal CPV phase $\mathcal{I} m\left(c_{b}\right)$ can be large, leading to observable deviations in the $B_{d, s}-\bar{B}_{d, s}$ mixing phases, but none in LMFV. Another example is $b \rightarrow s \nu \bar{\nu}$ and $s \rightarrow d \nu \bar{\nu}$ transitions. These receive contributions only from a single operator in Eq. (16) multiplied by the neutrino currents. Thus, new contributions to $B \rightarrow X_{s} \nu \bar{\nu}, B \rightarrow K \nu \bar{\nu}$ vs. $K_{L} \rightarrow \pi^{0} \nu \bar{\nu}, K^{+} \rightarrow \pi^{+} \nu \bar{\nu}$ are correlated in LMFV $\left(c_{b} \simeq c_{t}\right)$, see e.g., [10], but are independent in NLMFV with large $\tan \beta$. $O(1)$ effects in the rates would correspond to an effective scale $\Lambda_{\mathrm{MFV}} \sim 3 \mathrm{TeV}$ in the four fermion operators, with smaller effects scaling like $1 / \Lambda_{\mathrm{MFV}}$ due to interference with the SM contributions. Other interesting NLMFV effects involving the third generation, e.g., large deviations in $\operatorname{Br}\left(B_{d, s} \rightarrow \mu^{+} \mu^{-}\right)$and $b \rightarrow s \gamma$, arise in the MSSM at large $\tan \beta$, where resummation is required [11]. Contributions to $1 \rightarrow 2$ transitions which proceed through the charm $\left(c_{c}\right)$ and the top $\left(c_{t}\right)$ are correlated within $\operatorname{LMFV}\left(c_{t} \simeq c_{c} y_{t}^{2}\right)$, but are independent in the NLMFV case, even for small $\tan \beta$. Unfortunately, the smallness of the $c_{c}$ bilinear prevents tests of this correlation in the near future, e.g., via comparison of $K^{+} \rightarrow \pi^{+} \nu \bar{\nu}$ and the CPV decay $K_{L} \rightarrow \pi^{0} \nu \bar{\nu}$.

CP Violation. Assuming MFV, new CPV effects can be significant if and only if the UV theory contains new flavor-diagonal CP sources. The proof is as follows. If no flavor diagonal phases are present, CPV only arises from the CKM phase. In the exact $U(2)_{L}$ limit the CKM phase can be removed and the theory becomes $\mathrm{CP}$ invariant (at all scales). The only spurions that break the $U(2)_{L}$ flavor symmetry are $\phi_{u, d}$ and $\chi$. CPV in operators linear in $\chi$ is directly proportional to the CKM phase [cf. Eq. (16)]. Any additional contributions are suppressed by at least $\left[\phi_{u}^{\dagger} \phi_{u}, \phi_{d}^{\dagger} \phi_{d}\right] \sim\left(m_{s} / m_{b}\right)^{2}\left(m_{c} / m_{t}\right)^{2} \sin \theta_{C} \sim 10^{-9}$, and are therefore negligible.

Flavor diagonal weak phases in NLMFV can lead to new CPV effects in $3 \rightarrow 1$ and $3 \rightarrow 2$ decays. An example is $\Delta B=1$ electromagnetic and chromomagnetic dipole operators constructed from the first bilinear in Eq. (13). The operators are not Hermitian, hence their Wilson coefficients can contain new CPV phases. Without new phases, the untagged direct CP asymmetry in $B \rightarrow X_{d, s} \gamma$ would essentially vanish due to the residual $U(2)$ symmetry, as in the SM [12], and the $B \rightarrow X_{s} \gamma$ asymmetry would be less than a percent. However, in the NLMFV limit (large $y_{b}$ ), non-vanishing phases can yield significant CPV in untagged and $B \rightarrow X_{s} \gamma$ decays, and the new CPV in $B \rightarrow X_{s} \gamma$ and $B \rightarrow X_{d} \gamma$ would be strongly correlated. Supersymmetric examples of this kind were studied in [13], where new phases were discussed.

Next, consider the NLMFV $\Delta B=2$ effective operators. They are not Hermitian, hence their Wilson coefficients $\kappa_{i} / \Lambda_{\mathrm{MFV}}^{2}$ can also contain new $\mathrm{CP}$ violating phases. The operators can be divided into two classes: class-1, which do not contain light righthanded quarks $\left[\left(\overline{\tilde{d}}_{L}^{(2)} \chi \tilde{b}_{L, R}\right)^{2}, \ldots\right]$; and class-2, which do $\left[\left(\overline{\tilde{d}_{R}^{(2)}} \phi_{d}^{\dagger} \chi \tilde{b}_{L}\right)\left(\overline{\tilde{d}_{L}^{(2)}} \chi \tilde{b}_{R}\right), \ldots\right]$. Class-2 only contributes to $B_{s}-\bar{B}_{s}$ mixing, up to $m_{d} / m_{s}$ corrections. Taking into account that $S U(3)_{F}$ breaking in the bag parameters of the $B_{s}-\bar{B}_{s}$ vs. $B_{d}-\bar{B}_{d}$ mixing matrix elements is only at the few percent level in lattice QCD [14], we conclude that class-1 yields the same weak phase shift in $B_{d}-\bar{B}_{d}$ and $B_{s}-\bar{B}_{s}$ mixing relative to the $S M$. The 
class- 1 contribution would dominate if $\Lambda_{\mathrm{MFV}}$ is comparable for all the operators. For example, in the limit of equal Wilson coefficients $\kappa_{i} / \Lambda_{\mathrm{MFV}}^{2}$, the class- 2 contribution to $B_{s}-\bar{B}_{s}$ mixing would be $\approx 5 \%$ of class- 1 . The maximal allowed magnitude of CPV in the $B_{d}$ system is smaller than roughly $20 \%$. Quantitatively, for $\mathcal{I} m \kappa_{i} \approx 1$, this corresponds to $\Lambda_{\mathrm{MFV}} \approx 18 \mathrm{TeV}$ for the leading class1 operator, which applies to the $B_{s}$ system as well. Thus, sizable CPV in the $B_{s}$ system would require class- 2 contributions, with $O(1) \mathrm{CPV}$ corresponding to $\Lambda_{\mathrm{MFV}} \approx 1.5$ $\mathrm{TeV}$ for the leading class-2 operator. Conversely, barring cancelations, within NLFMV models NP CPV in $B_{s}-\bar{B}_{s}$ mixing provides an upper bound on $N P C P V$ in $B_{d}-\bar{B}_{d}$ mixing.

For $2 \rightarrow 1$ transitions the new CPV phases come suppressed by powers of $m_{d, s} / m_{b}$. All the $2 \rightarrow 1$ bilinears in (10), (11), (13), (14) are Hermitian with the exception of $\bar{d}_{L}^{(2)} \chi \chi^{\dagger} \phi_{d} \tilde{d}_{R}^{(2)}$. This provides the leading contribution to $\epsilon_{K}$ from a non-SM phase, coming from the operator $\mathcal{O}_{L R}=\left(\overline{\tilde{d}}_{L}^{(2)} \chi \chi^{\dagger} \phi_{d} \tilde{d}_{R}^{(2)}\right)^{2}$. Its contribution is $\approx 2 \%$ of the SM operator $\mathcal{O}_{L L}=\left(\tilde{\tilde{d}}_{L}^{(2)} \chi \chi^{\dagger} \tilde{d}_{L}^{(2)}\right)^{2}$ for comparable Wilson coefficients $\kappa_{L R, L L} / \Lambda_{\mathrm{MFV}}^{2}$. For $\kappa_{L L}, \mathcal{I} m \kappa_{L R} \approx 1$, a new contribution to $\epsilon_{K}$ that is $50 \%$ of the measured value would correspond to $\Lambda_{\mathrm{MFV}} \approx 5 \mathrm{TeV}$ for $\mathcal{O}_{L L}$ and $\Lambda_{\mathrm{MFV}} \approx 0.8 \mathrm{TeV}$ for $\mathcal{O}_{L R}$.

Note that the above new CPV effects can only be sizable in the large $\tan \beta$ limit. They arise from nonHermitian operators (such as the second operator in (15)), and are therefore of higher order in the $Y_{d}$ expansion. Whereas we have been working in the large $\tan \beta$ limit, it is straightforward to incorporate the small $\tan \beta$ limit into our formalism. In that case the flavor group is broken down to $U(2)_{Q} \times U(2)_{u} \times U(1)_{3} \times U(3)_{d}$ and the expansion in Eq. (3) no longer holds. In particular, resummation over $y_{b}$ is not required. Flavor violation is described by linearly expanding in the down type Yukawa couplings, from which it follows that contributions proportional to the bottom Yukawa are further suppressed beyond the SM CKM suppression.

Up quark sector. Finally we comment on the up sector. We work in the up-quark mass basis in which $\hat{\rho}_{u}=\hat{\rho}_{d}=0, \rho_{Q}=-\chi / 2, \phi_{u}=$ $\operatorname{diag}\left(m_{u}, m_{c}\right) / m_{t}$, while the flavor violating spurions are $\phi_{d}=V_{C K M}^{(2) \dagger} \operatorname{diag}\left(m_{d}, m_{s}\right) / m_{b}$ and $\chi=i\left(V_{u b}, V_{c b}\right)$. An important prediction of the NLMFV models for the upsector is that the new contributions are greatly enhanced for large $\tan \beta$. Consider top flavor changing neutral currents (FCNC). Within the SM they are highly suppressed by a combination of a loop factor, GIM and CKM suppression. This results in branching ratios $B R(t \rightarrow c X) \sim$ $\mathcal{O}\left(10^{-12}\right)$. An example of a FCNC bilinear operator in NLMFV is $\overline{\tilde{u}^{(2)}} \chi \tilde{t}$ [in the LMFV limit it corresponds to $\bar{c}_{L}\left(Y_{b} Y_{b}^{\dagger}\right)_{23} t_{L}$ and $\left.\bar{c}_{L}\left(Y_{b} Y_{b}^{\dagger}\right)_{23} y_{t} t_{R}\right]$. Model independent analysis shows that such an operator can lead to
$B R(t \rightarrow c X) \sim \mathcal{O}\left(10^{-5}\right)$ [15], which may be within the reach of the LHC.

Similar enhancements are expected for CPV in $D-$ $\bar{D}$ mixing. The relevant operators are $\left(\overline{\tilde{u}}_{L}^{(2)} \chi \chi^{\dagger} \tilde{u}_{L}^{(2)}\right)^{2}$ and $\left(\overline{\tilde{u}}_{L}^{(2)} \chi \chi^{\dagger} \tilde{u}_{L}^{(2)}\right)\left(\overline{\tilde{u}}_{L}^{(2)} \phi_{d} \phi_{d}^{\dagger} \tilde{u}_{L}^{(2)}\right)$. The resulting CP violation in mixing is estimated to be $\arg \left(M_{12} / \Gamma_{12}\right)=$ $O(5 \%)\left(1 \mathrm{TeV} / \Lambda_{\mathrm{MFV}}\right)^{2}(\sin 2 \gamma, \sin \gamma)$, respectively, where $\gamma=\arg \left(-V_{u d} V_{u b}^{*} / V_{c d} V_{c b}^{*}\right)$. For $\Lambda_{\mathrm{MFV}} \sim 1 \mathrm{TeV}$ this is four orders of magnitude greater than in the SM, and would be observable in the future. Operators of the type $\left(\overline{\tilde{u}_{L}^{(2)}} \chi \chi^{\dagger} \phi_{u} \tilde{u}_{R}^{(2)}\right)^{2}$ can contain a new CPV phase, but $\left(m_{c} / m_{t}\right)^{2}$ suppression renders them negligible by comparison. Unfortunately, experimental tests of MFV are generally very difficult in rare charm decays due to dominance of long-distance SM effects.

Concluding remarks. Above we focused on the formalism and low energy flavor violating observables. However, useful information can also be extracted from flavor diagonal quantities such as the new physics mass spectra [16] or non universal couplings to new gauge bosons [6]. For example, let us assume that new scalar states are in the fundamental of $U(3)_{u}$ so that the mass matrix squared is in its adjoint as in supersymmetric models (e.g. right-handed squarks, neglecting the mixing with left-handed squarks). Order one or larger splitting $\Delta m_{13}^{2}$ between the first two and the third generation would signal the NLMFV limit. Further insight would be provided by the mass spliting between the first two generations, $\Delta m_{12}^{2}$. In LMFV $\Delta m_{13}^{2}: \Delta m_{12}^{2}=m_{t}^{2}: m_{c}^{2}$, while in NLMFV this relation would receive large corrections from subleading expansions in the Yukawas. Finally we point out that NLMFV differs from the next-to-MFV (NMFV) [17] framework since the latter exhibits additional spurions at low energy.

Acknowledgements. We thank N. Arkani Hamed, J. Kamenik, D.E. Kaplan, A. Kronfeld, and A. Weiler for useful discussions, we also thank Y. Nir and especially S. Jag̈er for discussions and comments on the manuscript. A. K. is supported by DOE grant FG02-84-ER40153, G. P. by NSF grant PHY-06353354 and the Peter and Patricia Gruber Award, T. V. by DOE grant DE-FG0290ER40542.

[1] G. D'Ambrosio, G. F. Giudice, G. Isidori and A. Strumia, Nucl. Phys. B 645, 155 (2002) [arXiv:hep-ph/0207036].

[2] L. J. Hall and L. Randall, Phys. Rev. Lett. 65, 2939 (1990); R. S. Chivukula and H. Georgi, Phys. Lett. B 188, 99 (1987).

[3] A. J. Buras, P. Gambino, M. Gorbahn, S. Jager and L. Silvestrini, Phys. Lett. B 500, 161 (2001) [arXiv:hepph/0007085]; A. Ali and D. London, Eur. Phys. J. C 9, 687 (1999) [arXiv:hep-ph/9903535]; E. Gabrielli and G. F. Giudice, Nucl. Phys. B 433, 3 (1995) [Erratumibid. B 507, 549 (1997)] [arXiv:hep-lat/9407029]. 
[4] A. J. Buras, Acta Phys. Polon. B 34 (2003) 5615 [arXiv:hep-ph/0310208].

[5] For a recent discussion see e. g., P. Paradisi, M. Ratz, R. Schieren and C. Simonetto, arXiv:0805.3989 [hep-ph].

[6] A. L. Fitzpatrick, G. Perez and L. Randall, Phys. Rev. Lett. 100, 171604 (2008) [arXiv:0710.1869 [hepph]]; K. Agashe, G. Perez and A. Soni, Phys. Rev. D 71, 016002 (2005) [arXiv:hep-ph/0408134]; C. Csaki, A. Falkowski and A. Weiler, arXiv:0806.3757 [hep-ph].

[7] T. Feldmann and T. Mannel, Phys. Rev. Lett. 100 (2008) 171601 [arXiv:0801.1802 [hep-ph]].

[8] See e.g: S. Weinberg, Cambridge, UK: Univ. Pr. (1996).

[9] G. Colangelo, E. Nikolidakis and C. Smith, arXiv:0807.0801 [hep-ph].

[10] S. Bergmann and G. Perez, Phys. Rev. D 64, 115009 (2001) [arXiv:hep-ph/0103299]; C. Bobeth, M. Bona, A. J. Buras, T. Ewerth, M. Pierini, L. Silvestrini and A. Weiler, Nucl. Phys. B 726, 252 (2005) [arXiv:hep$\mathrm{ph} / 0505110]$.

[11] C. Bobeth, T. Ewerth, F. Kruger and J. Urban, Phys. Rev. D 66 (2002) 074021 [arXiv:hep-ph/0204225]; A. J. Buras, P. H. Chankowski, J. Rosiek and L. Slawianowska, Nucl. Phys. B 659 (2003) 3 [arXiv:hep- ph/0210145]; M. S. Carena, D. Garcia, U. Nierste and C. E. M. Wagner, Phys. Lett. B 499 (2001) 141 [arXiv:hep-ph/0010003]; G. Degrassi, P. Gambino and G. Giudice, JHEP 0012, 009 (2000) [hep-ph/0009337].

[12] J. M. Soares, Nucl. Phys. B 367, 575 (1991).

[13] T. Hurth, E. Lunghi and W. Porod, Nucl. Phys. B 704, 56 (2005) [arXiv:hep-ph/0312260]. [arXiv:hep-ph/0207241];

[14] D. Becirevic, V. Gimenez, G. Martinelli, M. Papinutto and J. Reyes, JHEP 0204, 025 (2002) [arXiv:heplat/0110091]; E. Gamiz, C. T. H. Davies, G. P. Lepage, J. Shigemitsu and M. Wingate, arXiv:0902.1815 [hep-lat].

[15] P. J. Fox, Z. Ligeti, M. Papucci, G. Perez and M. D. Schwartz, Phys. Rev. D 78, 054008 (2008) [arXiv:0704.1482 [hep-ph]].

[16] Y. Grossman, Y. Nir, J. Thaler, T. Volansky and J. Zupan, Phys. Rev. D 76, 096006 (2007) [arXiv:0706.1845 [hep-ph]].

[17] Z. Ligeti, M. Papucci and G. Perez, Phys. Rev. Lett. 97, 101801 (2006) [arXiv:hep-ph/0604112]; K. Agashe, M. Papucci, G. Perez and D. Pirjol, arXiv:hepph/0509117. 